\title{
Consolidation of HLW into Mineral-Like Materials by the SHS
}

\author{
Ruizhu Zhang ${ }^{1,2, ~ a, ~ Y o n g ~} \mathrm{Li}^{1, \mathrm{~b}}$, Yuxin Zhang ${ }^{1, \mathrm{c}}$ and Zhimeng Guo ${ }^{2, \mathrm{~d}}$, \\ Wanshan $\mathrm{Su}^{1, \mathrm{e}}$ \\ ${ }^{1}$ North China Institute of Water Conservancy and Hydroelectric Power, Zhengzhou,450045, China \\ ${ }^{2}$ Materials Science and Technology School, University of Science and Technology Beijing , Beijing \\ 100083, China \\ azhang820@sohu.com, 'liyong@ncwu.edu.cn, 'zhangyuxin@ncwu.edu.cn, 'zmguo@126.com, \\ 'sws1986@126.com
}

Keywords: Consolidation; Perovskite; High-Level Radioactive Waste(HLW); Self -Propagating High -Temperatures Synthesis(SHS).

\begin{abstract}
Samples of mineral-like materials based perovskite and model high-level wastes (strontium) were fabricated by the SHS-densification and tested. The results of MCC-1, XRD and SEM/EDS analysis show that the major phase is well concordant with the design. The samples were characterized by dense structure and fine chemical stability. It is a durable material with high-level waste loading and is in favor for final geological disposal.
\end{abstract}

\section{Introduction}

With the growing exploitation of nuclear energy, more and more radioactive wastes have been produced. In order to promote the exploitation of nuclear energy as well as to obtain sustainable growth in the exploitation of nuclear energy, it is very important to dispose the radioactive wastes that can lead to the nuclear pollution. One of the methods treating these materials is the consolidation of high-level radioactive waste (HLW) into inactive-towards-environment materials, such as glass, glass ceramics, and various mineral-like materials. One of the most pronounced disadvantages of glass is its thermodynamic instability, which appears during high-temperature crystallization caused by radioactive decay and is followed by a monolith destruction. SynRock[1,2], i.e., ceramics based on synthetic analogs for of titanate rock-forming minerals, is a more perfect material, which preserves its mechanical and chemical properties for a long time and can accept a large number of radioactive elements contained in high-level waste into the lattice.

The aim of this paper is to research the possibility and conditions of self-propagating high-temperature synthesis(SHS) of mineral-like perovskite-based ceramics containing model calcinates, and also to evaluate chemical stability of the obtained samples by leaching rates of Sr ions into water.

\section{Experimental}

Experimental principle. The mineral-like materials based on perovskite can be obtained by the SHS method according to the following chemical reaction equation:

$$
2 \mathrm{CrO}_{3}+3 \mathrm{Ti}+4 \mathrm{CaO}+\mathrm{TiO}_{2}+x \mathrm{SrO}=4 \mathrm{CaTiO}_{3}+\mathrm{xSrO}+2 \mathrm{Cr}+\mathrm{Q}
$$

thermit reaction equation:

$$
\mathrm{Fe}_{2} \mathrm{O}_{3}+2 \mathrm{Al}=2 \mathrm{Fe}+\mathrm{Al}_{2} \mathrm{O}_{3}+\mathrm{Q}
$$


where $\mathrm{SrO}$ is a model radioactive waster used in our work. The heat released from the reaction makes the reaction self-sustaining. Meanwhile, the waste melts, and the simulating nuclide $\left(\mathrm{Sr}^{2+}\right)$ is consolidated in the peroviskite.

thermit reaction enhance the absolute temperature $T_{a d}$, which lead to the reaction occurd in a liquid stage. As a resuct, the density and uniformity of product was improved with a decreased porosity. In addition, the loadage of the product was enhanced also.

Raw materials. The matrix was composed of $\mathrm{TiO}_{2}, \mathrm{CaO}, \mathrm{Ti}, \mathrm{CrO}_{3}, \mathrm{SrO}, \mathrm{Fe}_{2} \mathrm{O}_{3}$ and $\mathrm{Al}$ (see Table1). The nuclide ${ }^{90} \mathrm{Sr}$ was replaced by non-radioactive $\mathrm{Sr}^{2+}$, adding $\mathrm{wt}(\mathrm{SrO})=10 \% \sim 40 \%$ respectively. The green mixture components were mixed in a ball mill, according to the mole ratio with 2:3:4:1. The particle size of the milled powder was $50 \sim 70 \mu \mathrm{m}$. Pellets of $3 \mathrm{~kg}$ in weight, $25 \mathrm{~mm}$ in diameter, $20 \mathrm{~mm}$ in height were made from the green mixture by the method of cold pressing. The pellets were subject to self -propagating high -temperatures synthesis (SHS) densification[3,4].

Table 1 Element percent contents in mixture

\begin{tabular}{lccccccc}
\hline Materials & $\mathrm{CrO}_{3}$ & $\mathrm{Ti}$ & $\mathrm{CaO}$ & $\mathrm{TiO}_{2}$ & $\mathrm{SrO}$ & $\mathrm{Fe}_{2} \mathrm{O}_{3}$ & $\mathrm{Al}$ \\
\hline purity (\%) & 99 & 99.5 & $>97.5$ & 98.5 & $>95$ & $>95$ & $>95$ \\
piratical size(mesh) & -200 & -300 & -200 & -200 & -300 & -300 & -300 \\
\hline
\end{tabular}

Preparation of sample. The scheme of SHS consolidation is shown in Fig.1. After mixed, the matrix was pressed into a green compact, and then put it into the sands in a steel mold. A tungsten filament was contact with the compact and served as the igniting element. After pre-pressed, the compact was ignited and combusted from one end to another. Then full pressure was loaded to densify the synthesized ceramic material.

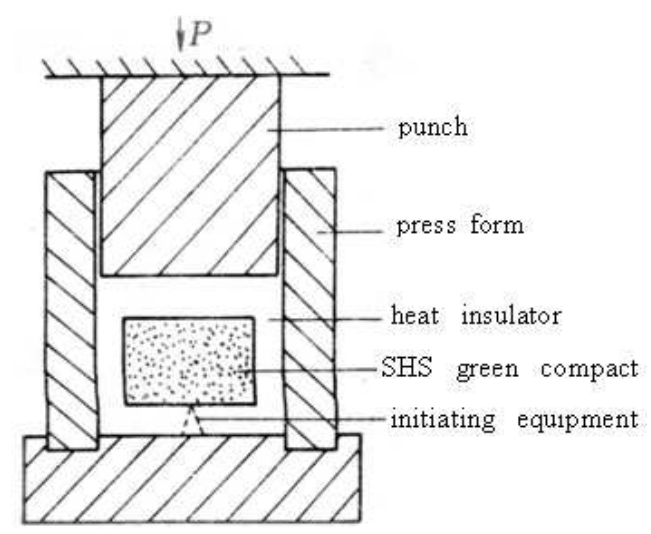

Fig.1 Scheme of SHS consolidation

Product analysis. The bulk density of the sample was measured using Archimedes liquid displacement technique. And its micro hardness (HV) was measured by micro hardness technique. The materials chemical stability was analyzed according to the international standard method (MCC-1) by leaching of $\mathrm{Sr}$ ions from the samples into distilled water at $\mathrm{T}=90{ }^{\circ} \mathrm{C} \quad 7$ days. Strontium(Sr) contents were determined using atomic absorption spectrophotometer. The microstructure of the product was observed using a scanning electron microscope (SEM. Cambridge S250 Mk2). phase composition was analyzed using Dmax-RB X-ray diffractometer ( $\mathrm{Cu} \mathrm{K} \alpha$ $\lambda=0.15406 \mathrm{~nm})$. And the microstructure of the product was observed using a scanning electron microscope (SEM. Cambridge S250 Mk2) 


\section{The analyzing of the results}

Physical properties. The five different content Perovskite mineral-like materials. We studied in this essay all have very good physical properties. The density $>4.2 \mathrm{~g} / \mathrm{cm}^{2}$, residual porosity $<0.2 \%$, microhardness $(\mathrm{HV})>1000 \mathrm{~kg} / \mathrm{mm}^{2}$ (see Table 2).

Table 2 Physical properties of Perovskite mineral-like materials

\begin{tabular}{ccccc}
\hline No & $\begin{array}{c}\mathrm{SrO} \\
(\mathrm{wt} \%)\end{array}$ & $\begin{array}{c}\mathrm{P} \\
\left(\mathrm{g} \bullet \mathrm{cm}^{-3}\right)\end{array}$ & $\begin{array}{c}\text { residual } \\
\text { porosity } \\
(\%)\end{array}$ & $\begin{array}{c}\text { microhardness } \\
(\mathrm{HV}) \\
\left(\mathrm{kg} \bullet \mathrm{mm}^{-2}\right)\end{array}$ \\
\hline 1 & 10 & 4.41 & 0.13 & 1070 \\
2 & 20 & 4.32 & 0.19 & 1045 \\
3 & 30 & 4.52 & 0.15 & 1038 \\
4 & 35 & 4.61 & 0.11 & 1089 \\
5 & 36 & 4.23 & 0.24 & 1060 \\
\hline
\end{tabular}

The chemical stability. The chemical stability of the materials obtained was determined by the rate of the $\mathrm{Sr}$ ion leaching form the samples into the distilled water at $90^{\circ} \mathrm{C}$. It was investigated from 7 day by the MCC-1 Static Leach rate test method described elsewhere [5]. The results of the Sr ion leaching from perovskite at $90^{\circ} \mathrm{C}$ as time dependence of the leaching rate(see Table 3).

Samples was found that the leaching rate in the compounds ratios was less than $2.1 \times 10^{-3} \mathrm{~g} / \mathrm{m}^{2} \bullet \mathrm{d}$ to the result of MCC-1. their leaching rates were less than $0.1 \times 10^{-1} \mathrm{~g} / \mathrm{m}^{2} \cdot \mathrm{d}$ (in boiling water), which were 2 or 3 orders less than that of the immobilized glass (12 \pm 0.4$)$. Addition, the leaching rates gradually decreased and finally reach a stable level, indicating that they could hold the high level radioactivity wastes in a stable way.

The method of consolidation in mineral-like materials is more widely used than other technologies of HLW neutralization. The data on leaching of Sr radio nuclides from glass[6,7], and their stable isotopes from perovskite obtained by the method of SHS densification $[\mathrm{wt}(\mathrm{SrO})=10 \% \sim 36 \%$ in a reactive mixture] are presented. It is show that leaching rate of element $\mathrm{Sr}$ is less than glass. perovskite can adapt to the variation of waste loading

Table3 MCC-1 Static Leach rate test results

\begin{tabular}{ccc}
\hline $\begin{array}{c}\text { Wasteform } \\
\text { material }\end{array}$ & $\begin{array}{c}\mathrm{Sr}^{2+} \text { loading/\% } \\
\mathrm{W} / \%\end{array}$ & $\begin{array}{c}\text { Leach } \mathrm{Rate} \mathrm{of}^{2+} / \\
\left(\mathrm{g} \bullet \mathrm{m}^{-2} \bullet \mathrm{d}^{-1}\right)\end{array}$ \\
\hline Provskite & 10 & $2.1 \times 10^{-3}$ \\
& 20 & \\
& 30 & \\
& 35 & $12 \pm 0.4$ \\
\hline
\end{tabular}

Note: Static Leach is the test method of Materials Characterization Center for chemical durability of nuclear waste wasteform sample, Test are in $90^{\circ} \mathrm{C}$ for $7 \mathrm{~d}$, using de-ionzing water as soak medium and surface area of sample to liquid volume equal to $20 / \mathrm{m} ; 90-19 / \mathrm{U}$ is atype of glass wasteform.

Component. An X-ray phase analysis of the products obtained by Fig.2 and Fig.3 Proved the formations of a perovskite structure with a pseudocubic crystal lattice. When calcinate was introduced into the green mixture, isomorphous substitution of $\mathrm{Ca}$ with $\mathrm{Sr}$ in the perovskite lattice was observed. The product obtained was a mixture of perovskite phasrs. 


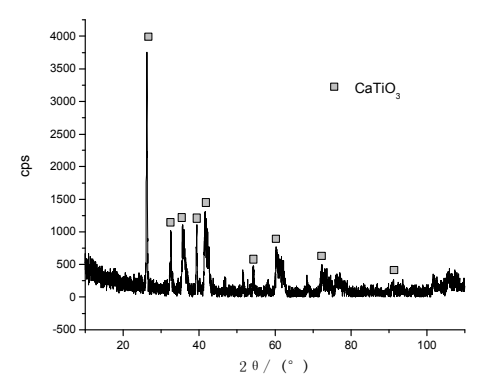

Fig.2 XRD pattern of matrix with wt $(\mathbf{1 0} \%)$ SrO in mass

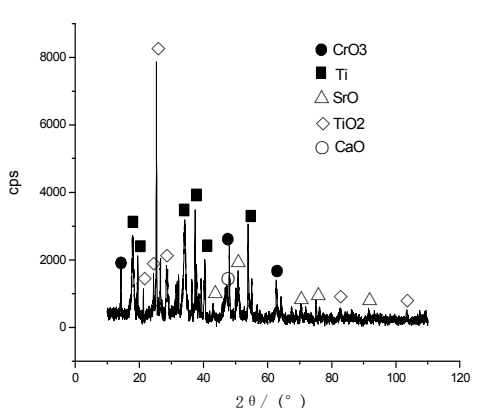

Fig.3 XRD pattern of dense sample with wt(10\%)SrO in mass

Microstructure of the densified sample. The microstructures of the perovskite-based materials obtained by the reaction are show in Figs. 4 . The perovskite phase is seen to be irregular gray tetragons (Fig4a,b ). The matrix completey encapsulates each separate block of perovskite, The grain size ranges from 15 to $25 \mu \mathrm{m}$. It proved that $\mathrm{SrO}$ is combined with $\mathrm{CaTiO}_{3}$ to form the uniform solid solution.

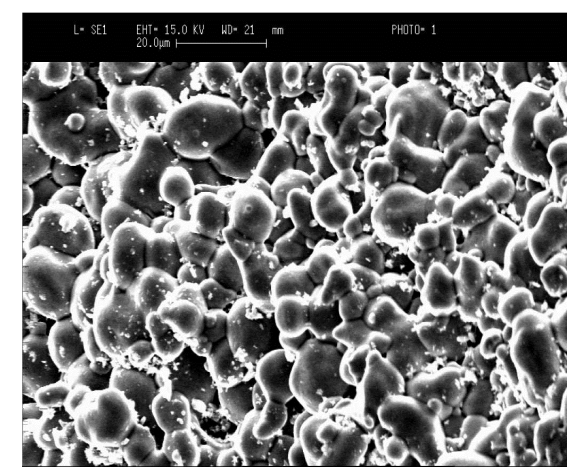

a fracture analysis diagram

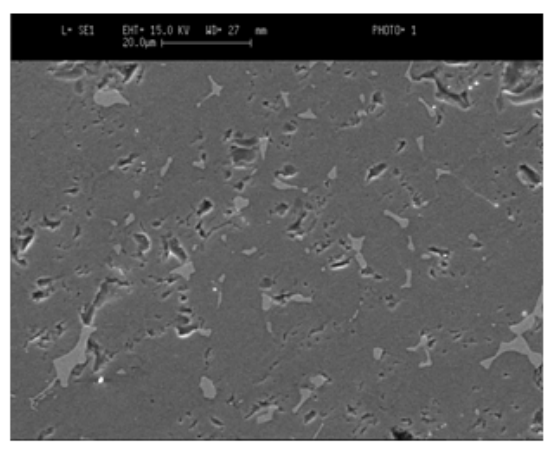

b Microstructure

Fig 4 Microstructure, Element distribution and EDX pattern of the dense sample with wt(10\%) SrO in mass

Measurement of the consolidation quantity of SrO. In the experiment, the addition amount of wt $(\mathrm{SrO})$ was $10 \%, 20 \%, 30 \%, 35 \%, 36 \%, 37 \%$ and $38 \%$ respectively. The reacted products were grinded into powders. The samples with various $\mathrm{SrO}$ content were characterized using SEM/EDS diffraction. It was found that the product is composed of single $\mathrm{CaTiO}_{3}$ phase when wt(SrO) content less than 37\% in Fig.5. But when the wt(SrO) content increase up to 37\%, free SrO appears in the product . It shows that consolidation limitation of $\mathrm{SrO}$ can be up to $36 \%$ with this SHS method using chromium oxide as an oxidant.

\section{Conclusion}

Perovskite mineral-like materials is a good vehicle for the consolidation of high level waste. Perovskite with SHS method show a food ability to consolidate $\mathrm{HLW}\left(\mathrm{Sr}^{2+}\right)$. Using chromium oxide as an oxidant can provide a higher temperature. the reaction is accomplished in liquid state, the solid solution can be formed in short time. SHS consolidation technique can increase the product density and improve well-distributed nature. Thus, Perovskite mineral-like materials is a durable material with high-level waste loading and is in favor for final geological disposal. 


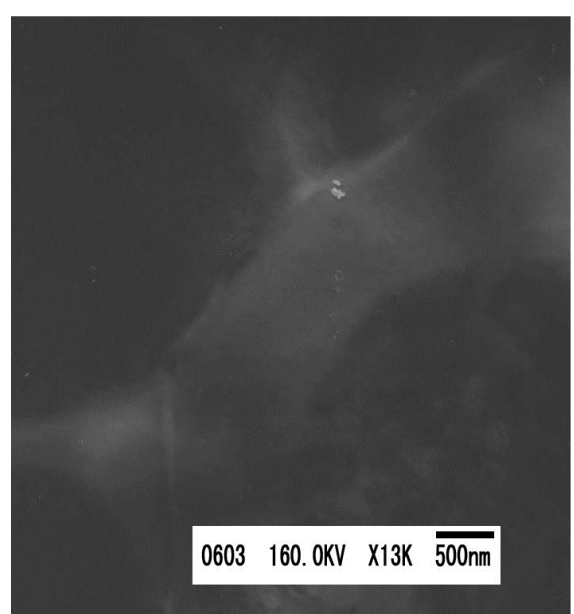

36wt\%SrO TEM

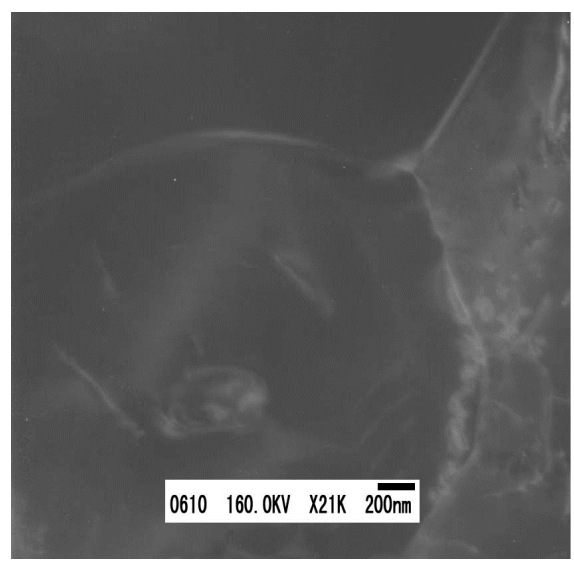

37wt\%SrO TEM

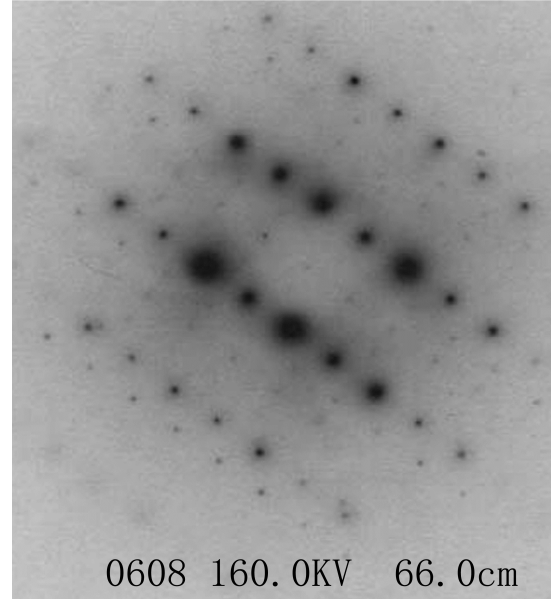

$36 w t \% \operatorname{SrO}$ EDS

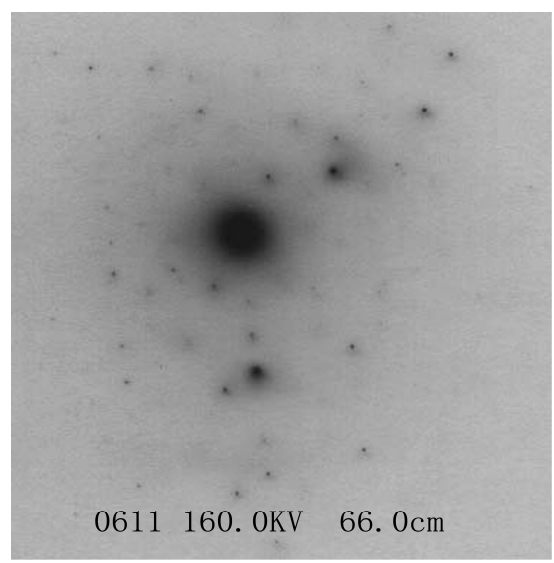

37wt\%SrO EDS

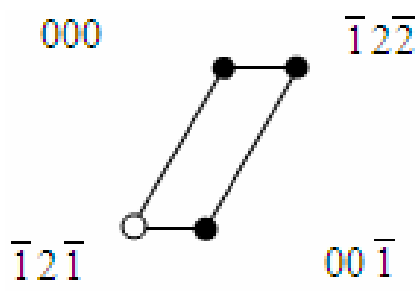

$\mathrm{CaTiO}_{3}: \quad \mathrm{B}=\mathrm{z}=[210]$

36wt\% EDS Calibration

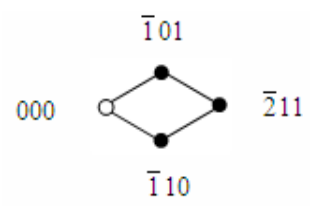

$\mathrm{CaTiO}_{3}: \mathrm{B}=\mathrm{z}=[111]$

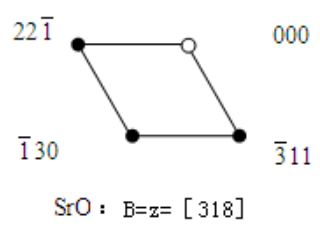

37wt\% EDS Calibration

Fig.5 TEM/EDS pattern of dense sample in mass

\section{References}

[1] Ringwood T.A., “A Rocky Graveyard for Nuclear,” J. New Sci., Vol. 99,1375 (1983), p. 756

[2] Zute W. and Ewing R., eds., Radioactive Waste Form for the Future, J. Elsevier Science Publ.B.V., (1988).

[3] Borovinskaya I.P., Barinova T.V., Ratnikov V.I., et al. Consolidation of Radioactives Waste into Mineral-like Material by the SHS Method,J. Inter.. SHS,Vol. 7,1 (1998), p.129-135

[4] Barinova T.V., Borovinskaya I.P., Ratnikov V.I., et al, SHS Immobilization of radioactive Wastes, J. Kay Engineering Materials,Vol. 217(2002), p.193 200

[5] Long -Term Leach Testing of Solidified Radioactive Waste Forms, C. Intl. Std. Reg. No.ISO 69, p.61-82

[6] Jiawei Sheng, Shanggeng Luo, Baolong Tang. High level Radioactive waste Vitrification and leaching behavior and its development J. Journal of the Chinese Ceramic society.Vol. 25,1(1997), p.83-88

[7] Egorov N.N. and Kudryavtsev E.G., "Regeneration and localization of RAW of a Nuclear Fuel Cycle,” J. Atomnaya, Vol. 74,4(1993), p.307-313 